\title{
PROCESO DE IDENTIFICACIÓN EN NIÑ@S VÍCTIMAS DE LA VIOLENCIA DE GÉNERO Y PILARES EN EL TRATAMIENTO
}

\author{
IDENTIFYING A PROCESS IN VICTIMS GIRLS/BOYS OF GENDER VIOLENCE \\ AND THE TREATMENT GUIDELINES
}

\author{
Natalia Ramirez S. ${ }^{1}$ \\ Universidad Autónoma de Madrid, España. \\ Universidad Nacional Mayor de San Marcos, Lima, Perú. \\ (RECIBIDO 7/5/2012; ACEPTADO 2/7/2012)
}

\begin{abstract}
RESUMEN
Trabajo sobre el proceso de identificación en niñas víctimas de la violencia de género y pilares en el tratamiento, con una visión teórica que examina la problemática desde aspectos conscientes e inconscientes; se incluyen dos ejemplos clínicos y se presentan investigaciones recientes que aportan datos relevantes a la temática.

Los objetivos se centran en conocer aspectos preventivos y de intervención más eficaces y poder contribuir, mediante la difusión de dicho saber, en el desarrollo saludable de menores víctimas de la violencia de género.
\end{abstract}

Palabras clave: Consciente, inconsciente, identificación, prevención, subjetividad, tratamiento violencia de género.

\begin{abstract}
It is about a process to identify the gender violence of girls/boys victims and the treatment guidelines as a theoretical view that examine the problem from conscious and unconscious aspects; there are two clinical examples and that are showed in recent researches that the matter gives us relevant data.

The objectives are centered in to know the preventive aspects and the most effective intervention to contributethrough the diffusion of that knowledge and the healthy development of gender violence in minor victims.
\end{abstract}

Keywords: Conscious, Unconscious, Identification, Prevention, Subjectivity, Treatment, Gender Violence.

1 docente de la facultad de psicología de la UNMSM. E-mail: tayle_03hotmail.com 


\section{INTRODUCCIÓN}

En 1996 la Asamblea Mundial de la Salud declara mediante una resolución (Resolución WHA 49.25) que la violencia es un problema de salud pública fundamental y creciente en todo el mundo, resalta las graves consecuencias de la violencia, tanto a corto como a largo plazo, para los individuos, las familias, las comunidades y los países, y recalca los efectos perjudiciales de la violencia en los servicios de atención de la salud. La OMS (2002) en el primer Informe Mundial sobre la violencia y la salud pide a los Estados Miembros que consideren urgentemente el problema de la violencia dentro de sus propias fronteras, y solicitó al director general de la Organización Mundial de la Salud que estableciera actividades de salud pública para abordar el problema.

La Organización Panamericana de la Salud define la violencia contra la mujer o basada en el género como "uno de los abusos contra los derechos humanos y uno de los problemas de salud pública más difundidos en el mundo actual (...) y es una manifestación extrema de la desigualdad de género que afecta a las mujeres y a los niñ@s (...) sus consecuencias son devastadoras y prolongadas y afectan la salud física y el bienestar mental".

Lo mencionado permite señalar que el mundo está reaccionando con preocupación ante el fenómeno de la violencia y que se están realizando y sugiriendo acciones para trabajar en los diferentes niveles de la prevención y promoción de la salud;diversas investigaciones indican que el fenómeno se mantiene y que lo efectuado hasta el momento intenta darle sostenibilidad a la reducción de la violencia de género que obstaculiza el desarrollo humano. Sin embargo, quisiera prestar especial relevancia al tema de los niñ@s víctimas de la violencia de género, un colectivo poco visibilizado pese a su mención en diferentes acuerdos internacionales, un colectivo que crece por las consecuencias negativas que tiene este fenómeno.

Un acercamiento a los contenidos de la violencia desde el género y la subjetividad, es poner nuestra atención en los procesos que van conformando las bases donde se sostiene la relación con uno mismo y con el otro; es escudriñar las condiciones históricas contingentes que posibilitan que ello se produzca. Es un proceso que deviene, y se configura referencialmente a través del otro. Octavio Paz (1969) afirma que la pregunta sobre nosotros se revela siempre como una pregunta sobre los otros.

Emilce Dío Bleichmar (1997), psiquiatra y psicoanalista, expresa que el género se convierte en una categoría valiosa de estudio desde las diferentes disciplinas, en la medida que incide en la constitución de la subjetividad.

Mi interés por la temática proviene de experiencias personales, académicas y profesionales, como psicóloga clínica y psicoterapeuta, mi labor me ha permitido confrontarme con una realidad teñida de violencia en muchos ámbitos, no solo que va en creciente aumento sino que la complejidad de la situación es cada vez más profunda. Planteo el trabajo con una visión teórica que examine la problemática desde una mirada diferente hacia aspectos no solo conscientes e individuales sino inconscientes y colectivos; asimismo incluyo dos ejemplos clínicos a manera de 
viñetas para ilustrar de manera práctica la exposición clínica de los casos y presento investigaciones recientes que aportan datos relevantes a la temática.

La violencia de género es un problema no solo considerado privado sino básicamente público y constituye uno de los símbolos que revelan las desigualdades existentes en nuestra sociedad. Considero que tanto el género como la subjetividad son categorías analíticas que permiten un acercamiento sucesivo a la heterogeneidad social, desde el fenómeno de la violencia y a este respecto destaco a un colectivo poco visibilizado, a los niñ@s víctimas de la violencia de género, que son objeto de diversos acuerdos internacionales y de estudios, con los que intentaré argumentar el proceso de identificaciónque siguen y los pilares en el tratamiento.

\section{Antecedentes}

Internacionalmente existen diversos acuerdos, normas y leyes que fundamentan la intervención en violencia de género entre los que están la Convención sobre la Eliminación de Todas las Formas de Discriminación contra la Mujer (CEDAW, 1979), Convención Interamericana para Prevenir, Sancionar, y Erradicar la Violencia contra la Mujer. Convención de Belem do Para (1994), Programa de Acción de la Conferencia de la Mujer, Beijing (1995); y, el Protocolo para prevenir, reprimir y sancionar la trata de personas especialmente de mujeres y niños (Protocolo de Palermo).

Particularmente en el tema de protección y atención a los niños encontramos que en el año 1989 se firma la Convención de los Derechos de los Niños, a los cuales muchos países se encuentran adheridos, y que pese a los grandes esfuerzos por darle cumplimiento aun se hace evidente las dificultades para asegurar los derechos básicos a los integrantes de este colectivo. Se hace necesario enfatizar que este acuerdo constituye el primer instrumento internacional que incorpora todos los derechos humanos: civiles, culturales, económicos, políticos y sociales.

Existen numerosos estudios llevados a cabo en los últimos años sobre las repercusiones físicas y emocionales de la violencia de género en las mujeres que lo padecen, sin embargo, son escasos en los casos de los menores víctimas, a pesar de que se admite lo vasto de las secuelas, teniendo constancia de que su desarrollo puede verse alterado en los ámbitos afectivo, emocional, cognitivo, social y físico y sabiendo que afecta a un aspecto imprescindible para el adecuado desarrollo de su personalidad, el sentimiento de seguridad y de confianza en el mundo y en las personas que lo rodean. Esto se ve agravado por el hecho de que el agresor es su propio padre, figura de referencia para el niñ@y de que la violencia ocurre dentro del hogar, lugar que debería ser de refugio y protección. Todo ello puede acarrear a corto plazo, la destrucción del sentimiento de seguridad, el aprendizaje de la indefensión, vivir bajo el temor y presentar emociones de ansiedad asociadas al hecho de que la experiencia traumática se pueda repetir en cualquier momento y, a largo plazo, el riesgo de reproducir lo presenciado en un futuro en sus relaciones de pareja, propiciando la transmisión generacional de la violencia de género. 
Luzón, Ramos, Recio y De la Peña (2011) citan el "Estudio sobre la violencia contra niños" de la Secretaría General de las Naciones Unidas (2006) donde resaltan que "la violencia dentro de la pareja hace aumentar el riesgo de violencia contra los niños en el seno de la familia". Asimismo mencionan que Pedreira (2003) describió el "Círculo interactivo de la Violencia Familiar"; una teoría según la cual considera que el inicio de la violencia familiar acontece con la violencia de género, y que más adelante suele generalizarse al conjunto de los integrantes de la familia debido a la alteración de los procesos vinculares paterno/materno- filiales.

\section{Algunas definiciones}

La violencia es una gran preocupación en el mundo por el aumento de la incidencia de los daños causados intencionalmente a personas de todas las edades y de ambos sexos, pero especialmente a las mujeres y a los niñ@s. Se entiende por violencia de género a "todo acto de violencia basado en el género que tiene como resultado posible o real un daño físico, sexual o psicológico, incluidas las amenazas, la coerción o la privación arbitraria de la libertad, ya sea que ocurra en la vida pública o privada" (Artículo 1 de la Declaración sobre la Eliminación de la Violencia contra la Mujer. Naciones Unidas, 1994).

Cuestionamientos hacia la aparición y el mantenimiento de la Violencia de Género nos conducen por miles de aristas, uno de esos caminos traslada a observar a los colectivos más vulnerables de padecerla y entre ellos a los niño@s quienes expectantes, van a sufrir sus consecuencias.

En su investigación, Luzón et al. señalaron que en la actualidad, se consideran menores expuestos a violencia de género en su ámbito familiar a todas los hij@s que viven en un hogar donde su padre o la pareja de su madre es violento contra la mujer; y, basándose en Corbalán y Patró (2003) estimaron que en torno a un $85 \%$ de estos menores presencian situaciones de violencia, es decir, son testigos directos de las agresiones a su madre: escuchan gritos, insultos, ruidos de golpes, ven las señales que dejan las agresiones, perciben el miedo y el estrés de la madre o están inmersos en el ciclo de la violencia. Cabe agregar que mencionan a la Academia Americana de Pediatría (AAP) para afirmar que ser testigo de violencia doméstica puede ser tan traumático para el niñ@ como ser víctima de abusos físicos o sexuales.

Crecer en una familia donde se da violencia de género, produce daño en los menores, tanto cuando presencian los acontecimientos violentos, como cuando, aun no presenciándolos, crecen y se desarrollan en estructuras familiares patriarcales. Así mismo, estas situaciones aumentan preocupantemente el riesgo de que los menores sean víctimas directas de abuso y malos tratos. Todo ello expone a estos al riesgo de padecer secuelas y daños, tanto a corto plazo como medio y largo en su historia de vida.

La Convención de Derechos de los Niños, aprobada por la Asamblea de la ONU, el 20 de noviembre del 1989 define el maltrato infantil como: "Toda forma de 
violencia, perjuicio o abuso físico o mental, descuido o trato negligente, malos tratos o explotación, mientras que el niño se encuentra bajo la custodia de sus padres, de un tutor o de cualquier otra persona que lo tenga a su cargo".

La existencia de patrones conductuales (mediadores afectivo-sociales estables), conformados en la infancia y retroalimentados a lo largo de la vida, mediatizan y predicen las conductas interpersonales en la vida adulta.

La violencia de género enlazada con la subjetividad nos lleva a introducirnos en el tema psicológico en su vertiente psicodinámica. Tanto Sigmund Freud como teorías posteriores en el psicoanálisis atribuyen al proceso de identificación un lugar prioritario en la estructura psíquica, el sujeto se constituye independiente como resultado de una serie de identificaciones que establece a lo largo de su vida.

Se reconoce que en la mayoría de las culturas la primera persona con la que tenemos una relación social íntima es una mujer: una madre o sus sustitutas o allegadas. Las teóricas feministas han sostenido que la represión, en especial la efectuada por los hombres, de estas relaciones primeras y los aspectos relacionales de nuestra subjetividad, son necesarias para la repetición de las culturas de dominio masculino.

\section{MÉTODO}

Según Barberá (1998) los dos tipos de cimientos sobre los que se construye la dimensión psicológica del género son los procesos biológicos de sexuación y los de asignación social. Esto no significa que esté predeterminada ni que exista una causalidad biunívoca entre fundamentación biológica o social y productos comportamentales de género. Los procesos psicológicos de identidad, aprendizaje de roles y construcción de esquemas estereotipados se desarrollan evolutivamente desde la concepción, por múltiples interacciones entre el individuo y su mundo particular, pudiendo llegar a tener una importancia relevante la manera como se razona, siente, enjuicia y se establece relaciones interpersonales.

El género se concibe como un conjunto de procesos, de naturaleza bio-psico-social que presenta las siguientes características: vinculación sexo - género, categorización social normativa, construcción subjetiva, sistema dinámica e interactivo; así como, contextualización teórica y cultural.

Se reconocen tres modelos para explicar el origen de las diferencias comportamentales: el sociológico o modelos evolucionistas, el del constructivismo social y el de la perspectiva psicodinámica. El primero centra el análisis en la evolución filogenética, el segundo se interesa por los orígenes socio-culturales cuya evolución y desarrollo deben insertarse en unas coordenadas históricas y culturales; y, el tercero, al que prestaré mayor atención, centra su estudio en el desarrollo a nivel individual, la ontogénesis de la psique y su acoplamiento a los principios y restricciones que impone la normativa social.

En la perspectiva psicodinámica, tanto Sigmund Freud como teorías posteriores atribuyen al proceso de identificación un lugar prioritario en la estructura psíquica, 
el sujeto se constituye independiente como resultado de una serie de identificaciones que establece a lo largo de su vida.

Sintetizando la posición freudiana el proceso de identificación primaria es similar pero no simétricos en varones y mujeres, durante la fase edípica la persona se identifica con determinados rasgos del progenitor del mismo sexo. El varón sustituye el objeto de la libido (madre) por el principio de la realidad (padre) y se identifica con rasgos de este último. En la mujer el proceso se complica, pues el primer objeto libidinal (madre) debe ser sustituido por el padre para volver de nuevo a la madre como modelo normativo a introyectar.

Retomando a Barberá (1998) expresa que muchas han sido las críticas al modelo freudiano y se ha llevado a replantear revisiones y proponer alternativas. Algunas inciden en la importancia de los factores de asignación social (Stoller, 1968); o bien afectan al desarrollo psicosexual de los varones, como el estudio del autodescubrimiento genital en el niño pequeño (Kleeman, 1966), o bien sobre la elaboración de los lazos de afecto y cooperación entre niños varones y padres (Kaftal,1991). Sin embargo, los aspectos más cuestionados son los de la concepción de la femineidad y el desarrollo psíquico de las mujeres. M. Klein (1932) plantea la hipótesis de la "femineidad primaria" como alternativa al criterio básico de la masculinidad de Freud.

Desde los presupuestos analíticos básicos, autoras como H. Deutsch (1946), C. Thompson (1943) o K. Horney (1967), que se interesaron por el psicoanálisis, en la medida que estudiaba la sexualidad y representaba un cuestionamiento a las normas, valores y concepciones establecidas; y, cuya participación fue alentada y valorada por el propio Freud, plantearon interrogantes buscando una nueva identidad y contribuyeron así a generar un debate crucial en el seno del psicoanálisis. Han enfatizado tanto la trascendencia que el rol materno ejerce sobre la vida de los niñ@s como la importancia que los factores sociales y culturales desempeñan en el proceso de identidad y en el aprendizaje de los roles de género.

Según afirma Flax (1995), para Freud la cultura exige a partir de la bisexualidad psicológica de ambos sexos, que uno de ellos adquiera una primacía: el hombre y la mujer no nacen ya sexuados, sino que devienen tales a través de su historia infantil, de sus relaciones intersubjetivas originarias en el seno de la cultura. Lo único que está definido en el momento del nacimiento es el sexo anatómico, pero no ocurre lo mismo ni con la posición subjetiva ni con su identidad sexual, producto de sus identificaciones y de la interiorización de ideales culturales relativos a la femineidad y a la masculinidad, ni con la orientación de su deseo sexual.

Todas las experiencias explícitas e implícitas que vivencien los niñ@s van a nutrir a esas primeras identificaciones, van a ir incorporándose en su repertorio mental, sea de manera negativa o positiva; lo que a su vez irá formando parte de su proceso de identidad. A este respecto menciona Bosch (2008) que la socialización es el proceso por el que aprendemos y hacemos nuestras las pautas de comportamiento social del medio socio-cultural en el que nos desenvolvemos, aprendemos e 
interiorizamos valores, actitudes, expectativas y comportamientos en interacción con otras personas.

Osborne (2009) cita al Dr. Marcos Rojas para referirse a la imposibilidad de la víctima para escapar de la tiranía de su verdugo en instituciones totales y que suelen darse en la intimidad del hogar. La familia se presenta como una opción para engendrar conflictos y contradicciones, se vende como un refugio seguro pero al mismo tiempo es el escenario donde se representan las más extremas pasiones humanas. En este caso en particular el hogar es un lugar donde se generan grandes tensiones porque es el espacio donde se despliega el propio yo, libre de mediaciones e inhibiciones sociales. Y en este punto los niñ@s como observadores van introduciendo en su sistema dichas relaciones entre los miembros de sus familias.

\section{Devenir de los niñas víctimas de la violencia de género}

De acuerdo a Díez y Vásquez (2006), las alteraciones y las consecuencias psicológicas y psicopatológicas a corto y largo plazo de niñ@s y adolescentes que han sufrido malos tratos y/o negligencia en la infancia y la adolescencia, varían según el momento evolutivo ( $0-3$ años, primera infancia, edad escolar, preadolescencia y/o adolescencia) en el que se produce el maltrato y de ahí que las consecuencias psicológicas difieran. En los primeros años, relacionado con el desarrollo del apego, se puede dar retraso del desarrollo motor, social, cognoscitivo y del lenguaje; retraso del crecimiento y desviaciones en el desarrollo y déficits intelectuales. En la primera infancia y en edad escolar, relacionado con el desarrollo socioemocional, aparecen dificultades de aprendizaje y trastornos comportamentales. En la adolescencia, etapa en la que existe un desarrollo asimétrico entre sus conocimientos y su experiencia se pueden idear soluciones utópicas, además que se abordan temáticas de la personalidad. Supone un importante desequilibrio en su desarrollo integral, y son frecuentes las conductas de huida del domicilio así como inicio de trastornos asociales. Las alteraciones psiquiátricas y psicopatológicas del maltrato incluyen ansiedad, conducta agresiva, ideación paranoide, trastorno por estrés postraumático, trastornos depresivos y riesgo de actuaciones suicidas. Se enfatiza que por lo general padecer malos tratos crónicos da lugar a conductas agresivas y violentas; así como que constituyen factores de riesgo para desarrollar conductas de maltratador en el futuro.

Luzón et al. clasificaron las secuelas en función a dos criterios: criterio de externalización-internalización, en donde los varones presentan con más frecuencia conductas agresivas y antisociales, mientras que las niñas más alteraciones emocionales relacionadas a la tristeza, queja somática o conductas de inhibición y miedo; y, el del peso a la transmisión generacional, esta última asociada al riesgo de reproducir lo vivenciado en el futuro en sus relaciones de pareja, a este respecto no existe un determinismo absoluto y las secuelas varían en función a las características propias del individuo (lo presenciado, la existencia o no de factores de protección y resilencia). 
Concluyeron en que existen secuelas psicopatológicas internas:

- Sintomatología depresiva caracterizada por sentimientos de tristeza, carencias afectivas, falta de autoestima, sentimiento de soledad, anhedonia y falta de reactividad a los estímulos habitualmente placenteros.

- Síntomas de ansiedad descritos como exceso de activación, síntomas de reexperimentación, comportamientos de evitación y labilidad emocional.

- Problemas de relación, inhibición y aislamiento como inhibición verbal, inseguridad, excesivo sentido del ridículo y el aislamiento y deseo de soledad.

Secuelas psicopatológicas externas denotando una mayor incidencia en chicos, posiblemente causado por la identificación con el rol paterno y los estereotipos de género, así tenemos:

- Búsqueda de atención caracterizada por conductas de llamada de atención, celos, fanfarroneo y presunción.

- Agresividad verbal cuyos indicadores son tendencia a discutir y a gritar, actitudes de terquedad e irritabilidad.

- Problemas de conducta o conductas disruptivas descrita como tendencia a trasgredir las normas y a discutir.

En cuanto a la transmisión generacional puntualizaron la existencia de:

- Sexismo interiorizado referido a la presencia de creencias sexistas vinculadas a la legitimación de la autoridad del padre lo que aumenta con la edad del menor que la padece.

- Percepción subjetiva de riesgo relacionado con la creencia que identificaran la violencia sin dificultad equiparándola a la fase de explosión, olvidando la paulatina instauración de la misma.

- Atribución causal donde tienden a justificar al agresor por pérdida de nervios, trastornos mentales y problemas o preocupaciones.

- Los varones tienden a normalizar el uso de la agresión verbal para resolver los conflictos a diferencia de las niñas.

\section{RESULTADOS}

\section{Caso N. ${ }^{\circ} 1$}

Niño de 6 años que acude a consulta acompañado de su madre y abuela paterna, refiriendo estas últimas, que el menor ha presenciado múltiples hechos de violencia verbal, física y emocional, por parte de su padre hacia él y su madre, ellos estuvieron radicando en Estados Unidos hasta hace unas semanas, tiempo en que la madre retorna a su país natal ayudada por los servicios de protección por violencia de género. Posterior a ello, han observado en el niño cambios en su 
carácter caracterizado por aislamiento social, rebeldía, gritos, llanto, negativas a conversar con su padre cuando este llama para saber de él.

\section{Comentario}

Observamos a un niño con secuelas psicopatológicas tanto internas como externas, siendo las primeras sentimientos de tristeza, de soledad, exceso de activación, labilidad emocional; y, las segundas, las mismas que se conocen tienen una mayor incidencia en varones, caracterizadas por conductas de llamada de atención, tendencia a gritar, actitudes de terquedad e irritabilidad.

\section{Caso N. ${ }^{\circ} 2$}

Adolescente de 12 años que acude a consulta en compañía de la madre debido a disfuncionalidad en la dinámica familiar por problemas de pareja con el padre. Ella presume que estas dificultades están afectando emocionalmente a la menor; actualmente, el padre se encuentra trabajando fuera de su zona de residencia y afirma que en los últimos años él ha estado maltratando psicológicamente a su hija con insultos y ofensas constantes, negando en alguna oportunidad su paternidad biológica, circunstancia poco entendible para la menor y generadora de rechazo afectivo hacia él. Académicamente la adolescente se encuentra con buenas calificaciones; sin embargo, la madre refiere que se ha presentado disminución en el desempeño de sus labores escolares, situación que no es aceptada por la progenitora puesto que constantemente le ha brindado mensajes de superación y evitación al fracaso; asimismo la describe como nerviosa y aislada de sus compañeras de colegio, permaneciendo sola en la casa hasta que ella retorna del trabajo.

\section{Comentario}

Se aprecia a una adolescente con secuelas psicopatológicas internas, atribuidas a las mujeres según investigaciones ya descritas en secciones anteriores, el caso clínico presenta como síntomas: sentimientos de tristeza, de soledad y disminución de su interés en actividades académicas, comportamientos de evitación y problemas de relación interpersonal.

\section{DISCUSIÓN}

Luzón et al. mencionan el informe elaborado en 2006 por Save The Children tras el estudio llevado a cabo en diversas comunidades autónomas de España sobre "atención a los niños y niñas víctimas de la violencia de género", y después de reconocer que la Ley Orgánica I/2004 de Medidas de Protección Integral contra la Violencia de Género ha favorecido los derechos de las mujeres en general y creado un marco teórico igualmente protector de los niñ@s víctimas de violencia de género, concluyeron que los recursos atencionales están en general descoordinados 
y no son suficientes ni adecuados para atender sus necesidades ni intervenirlos eficazmente.

Encontramos muchos programas de prevención y tratamiento, desarrollados para intervenir sobre estas consecuencias, principalmente en EEUU y Canadá, sin embargo, la evaluación de la efectividad de dichos programas es relativamente reciente por lo que el conocimiento sobre su eficacia no está suficientemente contrastado.

A partir del 2000, la planificación de intervenciones contiene una amplia gama de aspectos, no se centra en síntomas individuales sino que alberga áreas familiares, académicas, sociales e incluye el sistema de creencias. Se incentiva los modelos ecológicos y se incluye factores de riesgo y protección.

Los profesionales de Atención Primaria y Salud Mental han de tener una formación adecuada y específica que les permita la detección y seguimiento de las niñ@s víctimas de la violencia de género. El tratamiento debe incluir e integrar los aspectos pediátricos, sociales, legales, jurídicos y policiales, de investigación y de prevención. Los Protocolos y Guías de actuación sobre Maltrato (Atención al maltrato infantil desde el ámbito sanitario; Atención al maltrato infantil desde Salud Mental; Protocolos de Salud Mental Infantil para la Atención Primaria), que se llevan a cabo en diversas localidades, constituyen un punto de referencia y de obligado conocimiento para todos los profesionales de la salud.

Como menciona Navarro en el libro de Bosch (2008) las principales estrategias educativas de prevención - intervención que deberían estar incluidas dentro de programas formativos son:

- Trabajo cooperativo: Es una estrategia de enseñanza-aprendizaje basada en la influencia positiva y constructiva de la interacción social.

- Educación en valores: Es educar para una convivencia positiva basada en el respeto mutuo y mediante la interiorización de los principios democráticos que garantizan un compromiso colectivo donde todos participan en igualdad.

- Resolución de conflictos: la mediación: Es un proceso voluntario que implica confidencialidad, basado en el diálogo, en el que todas las partes han de realizar un esfuerzo por comunicarse, comprenderse y llegar a acuerdos justos.

- Orientación vocacional no sexista: Es un proceso de orientación dinámico y adaptado a cada estudiante, con sus intereses, aptitudes y valores particulares, con independencia de su sexo.

- Estrategias complementarias: Ampliar y mejorar la formación en diferentes ámbitos, es el desarrollo de talleres monográficos que pueden estar dirigidos al profesorado, como cursos de formación permanente; y luego aplicados a los alumnos en sesiones tutoriales.

- El trabajo debe incluir la labor terapéutica en los tres niveles de atención:

- Prevención Primaria: Con el objetivo de reducir factores de riesgo y aumentar factores de protección; mediante campañas de sensibilización, promoción de la 
salud (buen trato, aumento de la tolerancia a la frustración, alternativas al uso de la violencia), reducción de circunstancias ambientales que favorecen su aparición, entre otras.

- Prevención Secundaria: Dirigida a aquellos colectivos de alto riesgo, con mayor probabilidad que exhiban o desarrollen conductas violentas; mediante la detección y abordaje precoz de las situaciones de riesgo y promoción de la salud.

- Prevención Terciaria: Es el tratamiento de las víctimas y el entorno, promoción de la salud y competencia de las víctimas y de su entorno.

Los diversos modelos de intervención para este problema de salud pública nos invita a apreciar que: "Tanto las hipótesis psicoanalíticas como las que ofrecen los estudios de género conllevan una propuesta de transformación: en tanto el psicoanálisis brinda propuestas de transformación intrapsíquica acerca de la subjetividad femenina, los estudios de género se interrogan acerca de cuales son las condiciones de la producción sociohistórica de la subjetividad y sugieren recursos de transformación para esas condiciones" (Dio Bleichmar y Burin, 1999, p. 62).

Como señala Diez, J. y Vásquez, M. (2006), los mecanismos de negación, ocultación y represión se mantienen a lo largo del tiempo, aunque a veces la necesidad y el deseo de exteriorizar lo que ha permanecido oculto, siempre que las condiciones sociales o psicoterapéuticas lo permitan, se hace ineludible el recuerdo de un trauma, es en sí mismo traumático, ya que duele recordarlo. Pero rememorarlo a través de las palabras permite que el afecto reprimido se haga consciente, y posibilita una mayor integración de las víctimas con su pasado y con los acontecimientos traumáticos vividos. En este sentido, el objetivo terapéutico es que la víctima reconstruya su identidad sin el peso traumático de la violencia que se ha ejercido sobre ella, sin el rencor constante de haber sentido en su piel el odio de otros, que en muchos casos han sido figuras relacionales importantes en el desarrollo de los niñ@s.

Siguiendo a Luzón et al. un programa de tratamiento adecuado dirigido a menores que han sufrido exposición a violencia de género debe incluir:

- Profunda evaluación y valoración de la afectación del menor.

- Planificación de la intervención personalizada en función de los resultados de la evaluación.

Los programas podrán ser de naturaleza individual o grupal y siempre en coordinación con profesionales en caso de derivación, también con el profesorado, personal sanitario y la madre a fin de brindar pautas y darle seguimiento al caso.

Finalmente se propone elaborar protocolos de actuación personalizados para cada unidad familiar y que semanalmente se incorporen nuevos objetivos conductuales hasta conseguir cambios saludables en los implicados del caso. 


\section{CONCLUSIONES}

1. La violencia de género no es un problema del ámbito privado, es un elemento estructural del sistema patriarcal que nos invita a estudiarlo desde la esfera individual hasta la social, atravesando por factores de diversa índole y se dirige hacia los colectivos más vulnerables: mujeres y niñ@s.

2. Las diversas investigaciones exploran los efectos de la violencia de género en las mujeres, sin embargo son escasas las encontradas en niñ@s, a pesar de que se acepta la repercusión que tendrá dicha exposición en su desarrollo integral.

3. En un núcleo familiar donde se registra violencia de género se espera secuelas psicopatológicas internas y externas en los menores que la expectan, tanto a corto plazo como medio y largo en su historia de vida.

4. El estudio de la violencia de género y la subjetividad nos permite reconsiderar la importancia del proceso de identificación como un elemento prioritario para comprender la estructura psíquica de los seres humanos.

5. Los acuerdos internaciones concluyen que es necesario definir programas de intervención útiles que garanticen la seguridad del menor, donde cada implicado conozca su lugar; así también reforzar los vínculos entre los profesionales y trabajar en red social.

6. Las intervenciones terapéuticas incentivan modelos ecológicos, donde se incluye factores de riesgo y protección.

\section{ANÁLISIS PERSONAL}

El presente trabajo me ha permitido enlazar las variables violencia de género y subjetividad, asimismo apreciar los avances en investigación sobre los efectos en los menores expuestos a violencia de género y aspectos que se estiman más eficaces para la atención terapéutica.

Todas las experiencias que tengan los menores, objetivas y subjetivas, van a nutrir esas primeras identificaciones producto de sus relaciones vinculares con sus progenitores, y de esta manera serán, todas ellas, parte de su psiquismo, ya sea de manera positiva o negativa. Así esas vivencias iniciales unidas a la socialización, van a permitirles apropiarse de las pautas de comportamiento social del medio sociocultural en el que se desenvuelvan.

Creo que es oportuno subrayar que uno aprende e interioriza valores, actitudes, expectativas y comportamientos en interacción con otras personas. Y desde aquí la importancia de la prevención, para ofrecer ambientes saludables que favorezcan desarrollos igualmente saludables, en los miembros que los componen.

Finalmente me adscribo a las solicitudes de profesionales e instituciones diversas en demandar políticas públicas y acciones sostenidas entre la población y con ello para superar la desigualdad de género y transformar una sociedad machista 
y excluyente en una sociedad para tod@s, con la finalidad de avanzar hacia la igualdad en los ámbitos de la vida social, económica, cultural y política, pero que esto tendrían un mayor asidero si el trabajo considera el espacio de niñ@s y adolescentes.

\section{REFERENCIAS BIBLIOGRÁFICAS}

Barberá, E. (1998). Psicología del Género. España: Ariel.

Bosch, E. (2008). Violencia de Género. Algunas cuestiones básicas. España: Formación Alcalá.

Dio Bleichmar, E. (1997). La sexualidad femenina. De la niña a la mujer. Argentina: Paidós.

Dio Bleichmar, E. y Burin, M. (1999). Género, Psicoanálisis, Subjetividad. España: Paidós.

Flax, J. (1995). Psicoanálisis y feminismo. Pensamientos fragmentarios. España: Cátedra.

Informe Mundial de la Violencia y la Salud. Publicado en español por la OPS para la OMS. (2002). Washington.

Informe Violencia de Género 2006 Ministerio de Sanidad y Consumo. (2008). España.

Luzon, J., Ramos, E., Recio, P. y De la Peña, E. (2011). Impacto de la exposición a Violencia de Género en Menores. España : Instituto Andaluz de la Mujer. Consejería para la Igualdad y Bienestar Social. Junta de Andalucía.

Osborne, R. (2009). Apuntes sobre violencia de género. España: Bellaterra.

Paz, O. (1969). Posdata. México :Fondo de cultura económica.

Puleo, A. (2008). El reto de la igualdad de género. Nuevas perspectivas en Ética y Filosofía Política. España: Biblioteca Nueva.

Díez, J Y Vásquez, M. (2006). Consecuencias psicológicas y psicopatológicas del maltrato: Intervención desde atención primaria y salud mental. Recuperado de: http://hdl. handle.net/10401/3622

Domingo-Salvany, F. (2009). El maltrato infantil en atención primaria de salud 20 años después de la Convención de los derechos de los niños (1989 -2009). Pediatría Integral, XIII (10), 893-908. Recuperado de:http://www.sepeap.org/imagenes/secciones/ Image/_USER_Ped \%20Int \%2013-10\%20web.pdf\#page $=35$

www.observatorioviolencia.org/ 
